\title{
Jean-Yves Beziau
}

\section{TRIVIAL DIALETHEISM AND THE LOGIC OF PARADOX}

\section{Dedicated to Graham Priest for his 68th birthday}

\begin{abstract}
In this paper we explain that the paraconsistent logic LP (Logic of Paradox) promoted by Graham Priest can only be supported by trivial dialetheists, i.e., those who believe that all sentences are dialetheias.
\end{abstract}

Keywords: Dialetheia; Paraconsistent Logic; Many-Valued Logic; Truth

\section{Dialetheism and dialetheia}

Dialetheism is a philosophical position principally promoted by Graham Priest. According to him: "a dialetheia is a sentence $A$ such that both it and its negation $\neg A$ are true" and "Dialetheism is the view that there are dialetheias" [13]. An important point is to give concrete examples of dialetheias. Dialetheists often give as an example of dialetheia the sentence of the liar paradox.

Let us call relative dialetheist, someone who believes that some sentences are dialetheias and some other not and trivial dialetheist, the one who believes that every sentence is a dialetheia. Maybe one can find a good philosophical argument to defend trivial dialetheism, but it seems that most dialetheists, including Priest, are supporting relative dialetheism.

Editors' Note: The LLP editors are aware of the fact that some of Beziau's theses are challenging. However, our aim is to inspire the readers to discuss Priest's Logic of Paradox and its role in the paraconsistent framework. 
To be coherent a relative dialetheist should focus on systems of paraconsistent logic where some formulas are dialetheias and some other not. We point out in this paper that in the Logic of Paradox, LP, the main paraconsistent logic promoted by Priest, all formulas are dialetheias.

\section{The three-valued logic of paradox LP}

In 1979 [12] Priest has presented under the name LP (Logic of Paradox) a paraconsistent logic having the same truth-tables for negation, conjunction and disjunction as the three-valued logics L3 of Eukasiewicz [11] and K3 of Kleene [9]:

\begin{tabular}{|l||l|}
\hline & $\neg$ \\
\hline \hline 0 & 1 \\
\hline$\frac{1}{2}$ & $\frac{1}{2}$ \\
\hline 1 & 0 \\
\hline
\end{tabular}

\begin{tabular}{|c||c|c|c|}
\hline$\wedge$ & 0 & $\frac{1}{2}$ & 1 \\
\hline \hline 0 & 0 & 0 & 0 \\
\hline$\frac{1}{2}$ & 0 & $\frac{1}{2}$ & $\frac{1}{2}$ \\
\hline 1 & 0 & $\frac{1}{2}$ & 1 \\
\hline
\end{tabular}

\begin{tabular}{|c||c|c|l|}
\hline$\vee$ & 0 & $\frac{1}{2}$ & 1 \\
\hline \hline 0 & 0 & $\frac{1}{2}$ & 1 \\
\hline$\frac{1}{2}$ & $\frac{1}{2}$ & $\frac{1}{2}$ & 1 \\
\hline 1 & 1 & 1 & 1 \\
\hline
\end{tabular}

Defining $A \rightarrow B$ as $\neg A \vee B$, we get the truth-table of the implication of K3. The difference is that in LP the value $\frac{1}{2}$ is considered as designated and in L3 and $\mathrm{K} 3$ as non designated.

The logic LP has some strange features. LP has the same theorems as classical $\operatorname{logic}^{1}$, but it differs at the level of the consequence relation and also at the metalogical level - as we have proved in a previous paper, LP is not self-extensional [2].

We will concentrate here on the philosophical value of LP with regard to dialetheism.

\section{Every atomic formula of LP is a dialetheia}

In LP, if we restrict the name "true" to the value 1 , no formula is a dialetheia. This choice does not make sense for someone who is interesting in dialetheias.

On the other hand, if we use the name "true" for both designated values 1 and $\frac{1}{2}$, then any atomic formula $S$ is a dialetheia: according to

1 In particular in LP, $\neg(A \wedge \neg A)$ is a tautology. 
this matrix semantics, given an atomic formula $S$ there is a distribution of truth-values giving to it the value $\frac{1}{2}$. In the unique extension of this distribution into a valuation the value of the negation of this formula $\neg S$ is also $\frac{1}{2}$. Snow is white, $2+2=4, I$ am lying are therefore all dialetheias.

We have used here the word "formula" to stay neutral. Formulas can be interpreted as sentences, propositions or statements. Accordingly we may talk about sentential logic, propositional logic, or statement logic(cf. [10]). And if we want to stay neutral, we can talk about zero-order logic, a terminology that has been promoted especially in Poland (see e.g. [14]).

G. Priest and F. Berto write:

A dialetheia is a sentence, $A$, such that both it and its negation, $\neg A$, are true (we shall talk of sentences throughout this entry; but one could run the definition in terms of propositions, statements, or whatever one takes as one's favourite truth-bearer: this would make little difference in the context).

They want to stay neutral. When they are talking about " $A$ " and " $\neg A$ ", this shows that they have in mind formulas of a logical system (that can be interpreted as sentences, propositions, statements, etc.). ${ }^{2}$

$2+2=4$, whether considered as a sentence, a proposition or a statement cannot be decomposed (into respectively sentences, propositions, statements). This is why it is considered as atomic. The fact that $2+2=4$ can be false in classical zero-order logic may be surprising for someone who doesn't know this logic and its philosophy (logical atomism), mainly promoted by Wittgenstein. According to this perspective any atomic formula can be false: there are some distributions of truth-values giving falsity as truth-value to it. It can also be true according to some other distributions of truth-values. A distribution of truth-value was called by Wittgenstein a truth-possibilty (in German: Wahreitsmöglichkeiten; cf. [16]). Wittgenstein made a distinction between truth and logical truth he used the word "tautology" for logical truth. According to this theory which up to now is still the mainstream theory, only tautologies are always true and no atomic formula is a tautology. From the modern axiomatic viewpoint of arithmetics, it is not necessarily strange that $2+2=4$ can be false, because what is a tautology (in first-order classical

2 Few lines after they write: "Dialetheism amounts to the claim that there are true contradictions". We will not talk here about the ambiguous notion of true contradictions, we have recently written a paper on this topic [7] and see also [1]. 
logic) is a conditional molecular formula, $2+2=4$ being at the right of the conditional and on the left there is a conjunction of some axioms for arithmetics.

When going from two-valued logic to many-valued logic, there is the question to know how to call the additional values. in three-valued logic, the third value can be called "possible" or "undetermined", that was the original idea of Łukasiewicz. But anyway the set of truth-values has to be divided into two sets, the set of designated values and the set of non-designated values, in order to define the notions of logical truth and consequence relation. In LP, as we have explained, the third value is considered as designated. This is already a good reason to consider it as "truth" (together with the other designated value). Furthermore this is the only way to consider that a formula and its negation can both be true according to the same valuation.

Another option would be to consider that a dialetheia is a formula $A$ such that $A$ and $\neg A$ can, not necessarily according to the same valuation, both be true. But in this case all atomic formulas of classical zeroorder logic are dialetheias, since an atomic formula can sometimes be true, sometimes be false and therefore, its negation can also sometimes be true. In this case non-dialetheias can only be molecular formulas. Classical logic could work to support (relative) dialetheism, no need to use a paraconsistent logic.

\section{Every molecular formula of LP is a dialetheia}

Let us now prove the following rather trivial theorem:

Theorem 1. In LP calling "true" both designated values, every molecular formula is a dialetheia.

Proof. Consider a molecular formula $M$. There is at least one distribution of truth-values giving to all its atomic formulas the value $\frac{1}{2}$. The unique extension of this distribution to a valuation obviously gives the value $\frac{1}{2}$ to $M$ and also to $\neg M$.

As a corollary, we see that there are not antilogies in LP, i.e., formulas which are always false, i.e., having always the value 0 . 


\section{LP, relative and trivial dialetheisms}

What has been pointed out in this paper, i.e., that any formula in LP is a dialetheia, is not something against LP or dialetheism, it only shows that LP is not compatible with relative dialetheism. The relative dialetheist has to look for another system of paraconsistent logic.

In most of the known systems of paraconsistent logic, all atomic formulas are dialetheias (for a general view of paraconsistent logic; see e.g. [8]). So these systems cannot support relative atomic dialetheism. In these systems Snow is blue, $0+0=0, I$ am telling the truth are all dialetheias. This is not necessarily a problem. It is the case of the paraconsistent logic $\mathrm{Z}$ that we have previously developed (cf. [4]; Z is equivalent to $\mathrm{S} 5$, see [3]). In this logic, there are molecular formulas which are not dialetheias, these are in fact exactly the tautologies and the antilogies. This is fairly reasonable.

There are other cases: systems of paraconsistent logic where all atomic formulas are dialetheias but where there is not a single molecular formula which is a dialetheia. This is the case for example of the logic P1 of Antonio Mário Antunes Sette [15]. From a mathematical point of view, many constructions are indeed possible. It is worth to develop philosophical ideas supporting these constructions. And viceversa: starting from some philosophical ideas, it is interesting to develop mathematical constructions in harmony with them.

One can have some good philosophical motivations to support absolute trivial dialetheism (i.e., both atomic and molecular formulas are dialetheias), but LP is not necessarily the best logic to support this philosophical option, and in fact it was not developed in this perspective. De Morgan logic can be more interesting. ${ }^{3}$

\section{References}

[1] Becker Arenhart, J. R., "Liberating paraconsistency from contradiction", Logica Universalis, 9 (2015): 523-544. DOI:10.1007/s11787-015-0131-y

[2] Beziau, J.-Y., "Idempotent full paraconsistent negations are not algebraizable", Notre Dame Journal of Formal Logic, 39, 1 (1998): 135-139. DOI: $10.1305 / \mathrm{ndjfl} / 1039293025$

[3] Beziau, J.-Y., "S5 is a paraconsistent logic and so is first-order classical logic", Logical Investigations, 9 (2002): 301-309.

3 About De Morgan logic see [5] and [6]. 
[4] Beziau, J.-Y., "The paraconsistent logic Z. A possible solution to Jaśkowski's problem", Logic and Logical Philosophy, 15 (2006): 99-111. DOI : 10.12775/LLP.2006.006

[5] Beziau, J.-Y., "Bivalent semantics for De Morgan logic (the uselessness of four-valuedness)", pages 391-402 in W. A. Carnielli, M.E. Coniglio, and I. M. L. D'Ottaviano (eds.), The many sides of logic, College Publication, London, 2009.

[6] Beziau, J.-Y., "History of truth-values", pages 233-305 in D. M. Gabbay, F. J. Pelletier, and J. Woods (eds.), Handbook of the History of Logic, Vol. 11 "Logic: A history of its central concepts", Elsevier, Amsterdam, 2012.

[7] Beziau, J.-Y., "Round squares are no contradictions (Tutorial on Negation, Contradiction and Opposition)", in J.-Y. Beziau, M. Chakraborty, S. Dutta (eds.), New Directions in Paraconsistent Logic, Springer, New Delhi, 2016.

[8] Beziau, J.-Y., W.A. Carnielli, and D. M. Gabbay (eds.), Handbook of Paraconsistency (Studies in Logic), College Publication, London, 2007.

[9] Kleene, S., "On a notation for ordinal numbers", Journal of Symbolic Logic, 3 (1938): 150-155. DOI : 10.2307/2267778

[10] Klement, K., "Propositional logic", Internet Encyclopedia of Philosophy, 2015. http://www. iep.utm.edu/prop-log/

[11] Łukasiewicz, J., "O logice trójwartościowej", Ruch Filozoficzny, 5 (1920): 170-171.

[12] Priest, G., "The logic of paradox", Journal of Philosophical Logic, 8, 1 (1979): 219-241. DOI : 10.1007/BF00258428

[13] Priest, G., and F. Berto, "Dialetheism", Stanford Encyclopedia of Philosophy, 2013. http://plato.stanford.edu/entries/dialetheism/

[14] Pogorzelski, W. A., Notions and theorems of elementary formal logic, Warsaw University, Białystok Branch, Białystok, 1994.

[15] Sette, A.M., On the propositional calculus P1, "Notas e comunicacões de matemática", Vol. 17, Recife, 1971.

[16] Wittgenstein, L., "Logisch-philosophische Abhandlung", Annalen der Naturphilosophie, 14 (1921), 185-262. Translated as Tractatus LogicoPhilosophicus, Kegan Paul, London, 1922.

JEAN-YVES BEZIAU

University of Brazil, Rio de Janeiro

and Visiting Professor, University of California, San Diego

(supported by the CAPES grant BEX 2408/14-07)

Brazilian Research Council, CNPq

jyb@jyb-logic.org 